Check for updates

Cite this: RSC Adv., 2017, 7, 45852

Received 19th July 2017

Accepted 20th September 2017

DOI: 10.1039/c7ra07949d

rsc.li/rsc-advances

\section{A RGB random laser on an optical fiber facet}

\author{
Tianrui Zhai, (D)* Lianze Niu, Fengzhao Cao, Fei Tong, Songtao Li, Meng Wang \\ and Xinping Zhang $\mathbb{B D}^{*}$
}

A red-green-blue (RGB) random laser consisting of three polymer layers and two isolated layers is fabricated on an optical fiber end facet by a dip-coating method. The total thickness of the laser device is approximately $3600 \mathrm{~nm}$. Silver nanoparticles are embedded in each of the three polymer layers and provide strong plasmonic enhancement of the pump light. Simultaneous RGB plasmonic random lasing is observed within the multilayer structure located on the fiber end facet under pulsed optical pumping conditions. The results reported here can be used directly in the design of compact laser sources and laser-based sensors.
Over the past decade, plasmonic random lasers have attracted considerable attention because of their great potential for use as display and sensing devices. ${ }^{1-8}$ The lasing threshold of a plasmonic random laser is much lower than that of a conventional random laser because of its large scattering cross-section and the localized surface plasmon resonance of the metal nanoparticles (NPs) used in the structure. ${ }^{9-13}$ Additionally, red-greenblue (RGB) plasmonic random lasers have been studied as potential multicolor output devices. ${ }^{14-16}$ Recently, the use of a combination of random lasers and optical fibers has been shown to improve the miniaturization of laser sources. ${ }^{17-24}$ The directionality of the random laser emissions can be improved significantly by the waveguide effect of the optical fiber. ${ }^{25,26}$ These random fiber lasers therefore have great potential for a wide range of applications including sensing, medicine, and telecommunications. However, integration of the RGB plasmonic random laser structure on the tip of an optical fiber, which would meet requirements for the development of compact integrated plasmonic random lasers, has not been explored to date.

In this work, an RGB plasmonic random laser consisting of three polymer layers separated by two polyvinyl alcohol (PVA) spacers is fabricated directly on the optical fiber end facet by a dip-coating method. The proposed configuration can be regarded as a simple superposition of three independent lasers because of the waveguide effect. The total thickness of the fivelayer structure is approximately $3600 \mathrm{~nm}$. The polymer layers are doped with silver (Ag) NPs to provide plasmonic enhancement of the pump light. Low-threshold RGB random lasing is observed simultaneously when the pump power exceeds the threshold of the laser device in the optical pumping

Institute of Information Photonics Technology and College of Applied Sciences, Beijing University of Technology, Beijing 100124, China. E-mail: trzhai@bjut.edu.cn; zhangxinping@bjut.edu.cn experiments. This approach offers a new way to fabricate compact multicolor fiber laser sources.

The active materials used in this experiment are three typical light-emitting polymers: poly[9,9-dioctylfluorenyl-2,7-diyl] endcapped with dimethyl phthalate (PFO, American Dye Source), poly[(9,9-dioctylfluorenyl-2,7-diyl)-alt-co-(1,4-benzo-(2,1',3)-thiadiazole)] (F8BT, American Dye Source), and poly[2-methoxy5-(3', $7^{\prime}$-dimethyloctyloxy)-1,4-phenylenevinylene] (MDMO-PPV, Sigma-Aldrich). PFO, F8BT, and MDMO-PPV were all dissolved in xylene with concentrations of $12.5,23.0$, and $8.5 \mathrm{mg} \mathrm{ml}^{-1}$, respectively. The Ag NPs used as dopants in the polymer solutions were prepared using a one-step synthesis method ${ }^{27}$ and were also dissolved in xylene with a concentration of $4.0 \mathrm{mg} \mathrm{ml}^{-1}$. The $\mathrm{Ag}$ NP solution was then mixed with an equal volume of polymer solution. The mixed solution was then oscillated ultrasonically for $15 \mathrm{~min}$ to guarantee its homogeneity. The PVA (PVA 107, Celanese Chemicals, Germany) spacer was dissolved in deionized water at a concentration of $40.0 \mathrm{mg} \mathrm{ml}^{-1}$. The optical fiber, which has a core diameter of $600 \mu \mathrm{m}$ and is $10 \mathrm{~cm}$ long, acts as the substrate. The RGB plasmonic random laser was fabricated on the end face of the optical fiber by a dip-coating method. One fiber end face was dipped into the mixed solution containing the F8BT polymer and the Ag NPs, and then dried at room temperature. As a result, an F8BT layer containing embedded Ag NPs was fabricated on the fiber facet. The fiber was then dipped into the PVA solution to form a PVA coating over the F8BT layer. The crucial factor here is that the difference between the solvents of the polymer and the PVA ensures the immiscibility of the adjacent layers. A MDMOPPV layer, a PVA layer, and a PFO layer were then fabricated in a similar manner on the double layer (PVA/F8BT) structure in sequence. As a result, a five-layer structure was constructed on the fiber facet to form the RGB plasmonic random laser.

Fig. 1 shows a schematic diagram of the RGB plasmonic random laser on the fiber tip. A 150 fs laser is used as the pump 


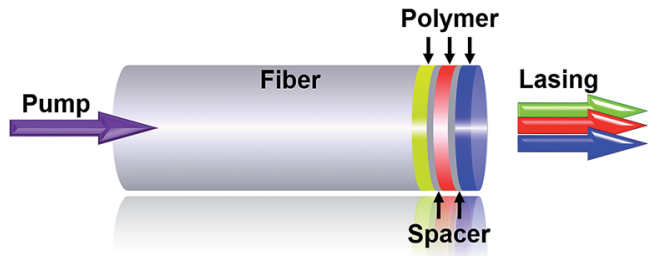

Fig. 1 Schematic of RGB plasmonic random laser formed on an optical fiber end facet. Black arrows indicate the five layers of the structure. The purple arrow indicates the pump beam direction. The RGB arrows denote the random laser output from the fiber tip.

source. The pump beam is coupled into one end of the fiber to excite the RGB random laser at the opposite end, as shown in Fig. 1. The purple arrow indicates the direction of the pump beam. The RGB arrows denote the plasmonic random laser output from the optical fiber facet.

In the experiments, the green, red, and blue random laser layers were fabricated on the fiber facet in sequence. Microscope photographs of these laser devices are shown in Fig. 2. Fig. 2(a) and (d) show the green random laser layer located on the fiber tip. The green and red random laser layers on the fiber facet are then shown in Fig. 2(b) and (e). Finally, the RGB random laser layers on the fiber facet are shown in Fig. 2(c) and (f). The F8BT, MDMO-PPV, PFO, and PVA layer thicknesses were $2000 \mathrm{~nm}, 400 \mathrm{~nm}, 800 \mathrm{~nm}$, and $200 \mathrm{~nm}$, respectively. All these thicknesses were optimized after a series of experiments was performed. The refractive indices of the F8BT, MDMO-PPV, and PFO layers and the optical fiber are 1.94 (at $\lambda=572 \mathrm{~nm}$ ), 1.66 (at $\lambda=638 \mathrm{~nm}), 1.78($ at $\lambda=466 \mathrm{~nm})$, and 1.54 (at $\lambda=572 \mathrm{~nm}$ ), respectively.

The refractive indices of the PVA layers are 1.72, 1.55, and 1.46 at 466, 572, and $638 \mathrm{~nm}$, respectively. These three wavelengths represent the emission peaks of the RGB random laser and will be discussed in detail later. Three polymer waveguides thus exist within the five layer structure. The RGB random lasing is a result of multiple instances of scattering of the photoluminescence (PL) of the polymers at the interfaces between the disordered plasmonic Ag NPs and the polymers, and this lasing is enhanced by the polymer waveguide because of the confinement of the scattered light. The thicknesses and the refractive indices of the five films were measured using an optical interferometric profiler (NanoMap 1000 WLI, AEP Technology) and a spectroscopic ellipsometer (ESNano, Ellitop), respectively.

The morphologies of the Ag NPs on the glass substrate and those embedded in the polymer were measured by scanning electron microscopy (SEM; Hitachi S-4800), as shown in the images in Fig. 3(a) and (c). Fig. 3(b) shows an SEM image of the $\mathrm{Ag}$ NPs that indicates that the typical Ag NP diameter is approximately $130 \mathrm{~nm}$. Fig. 3(d) shows the extinction spectra of the Ag NPs and the polymer films with embedded Ag NPs as measured using a spectrophotometer (U-4100, Hitachi). The black curve represents the extinction spectrum of the Ag NPs, which shows a peak at $426 \mathrm{~nm}$. The full width at half maximum (FWHM) value of the extinction spectrum of Ag NPs exceeds $40 \mathrm{~nm}$; this is attributed to the localized surface plasmon resonance of the $\mathrm{Ag}$ NPs. The pump beam wavelength is $400 \mathrm{~nm}$, as indicated by the purple line in Fig. 3(d). The figure shows that the plasmonic resonance peak of the Ag NPs overlaps with the pump wavelength. This indicates that the Ag NPs in the multilayered structure play a major role in the pump enhancement required for RGB random lasing. The dark purple curve represents the extinction spectrum of the three polymer films (F8BT, MDMOPPV, and PFO) with the embedded Ag NPs and can be regarded as a linear superposition of the spectra of the three components. The extinction spectrum of the multilayered structure is thus very broad and has a linewidth of more than $220 \mathrm{~nm}$ at FWHM. One of its extinction peaks overlaps with the pump wavelength, as shown in Fig. 3(d), and this enhances the pump efficiency.

The femtosecond laser that was used as the pump source during the spectral measurements has a wavelength of $400 \mathrm{~nm}$, a repetition frequency of $1 \mathrm{kHz}$, and maximum output power of $40 \mathrm{~mW}$. The pump beam was passed through a neutral optical
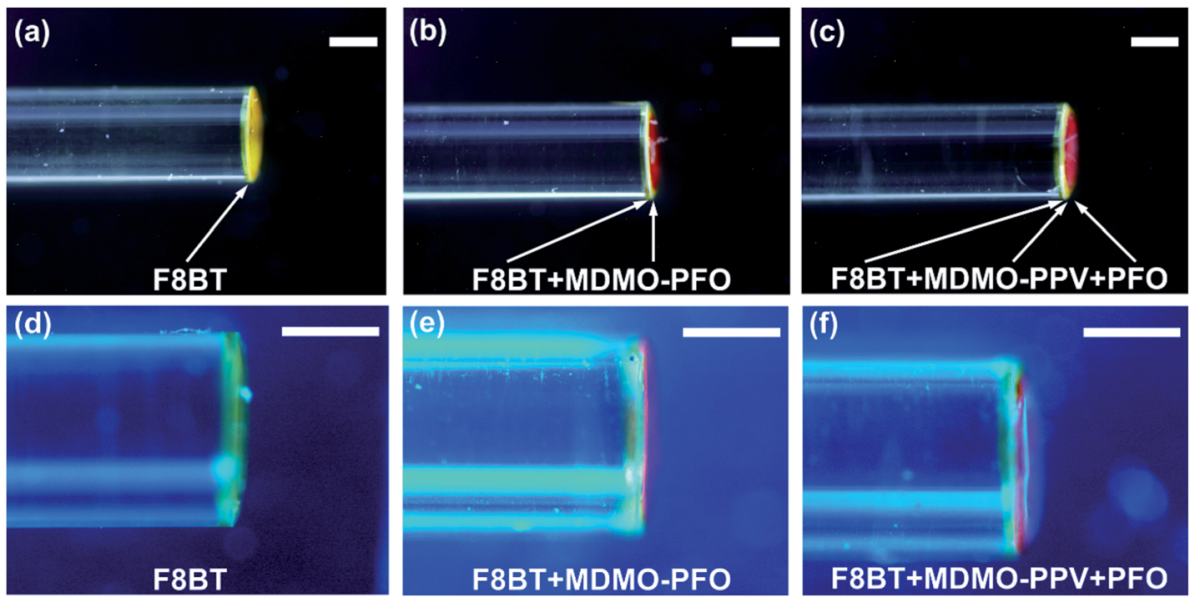

Fig. 2 Microscope photographs of the plasmonic random laser on the fiber facet at different magnifications. (a) and (d) Show the green random laser on the fiber tip. (b) and (e) Show the green and red random lasers on the fiber tip. (c) and (f) Show the RGB random lasers on the fiber tip. The scale bar represents $300 \mu \mathrm{m}$. 

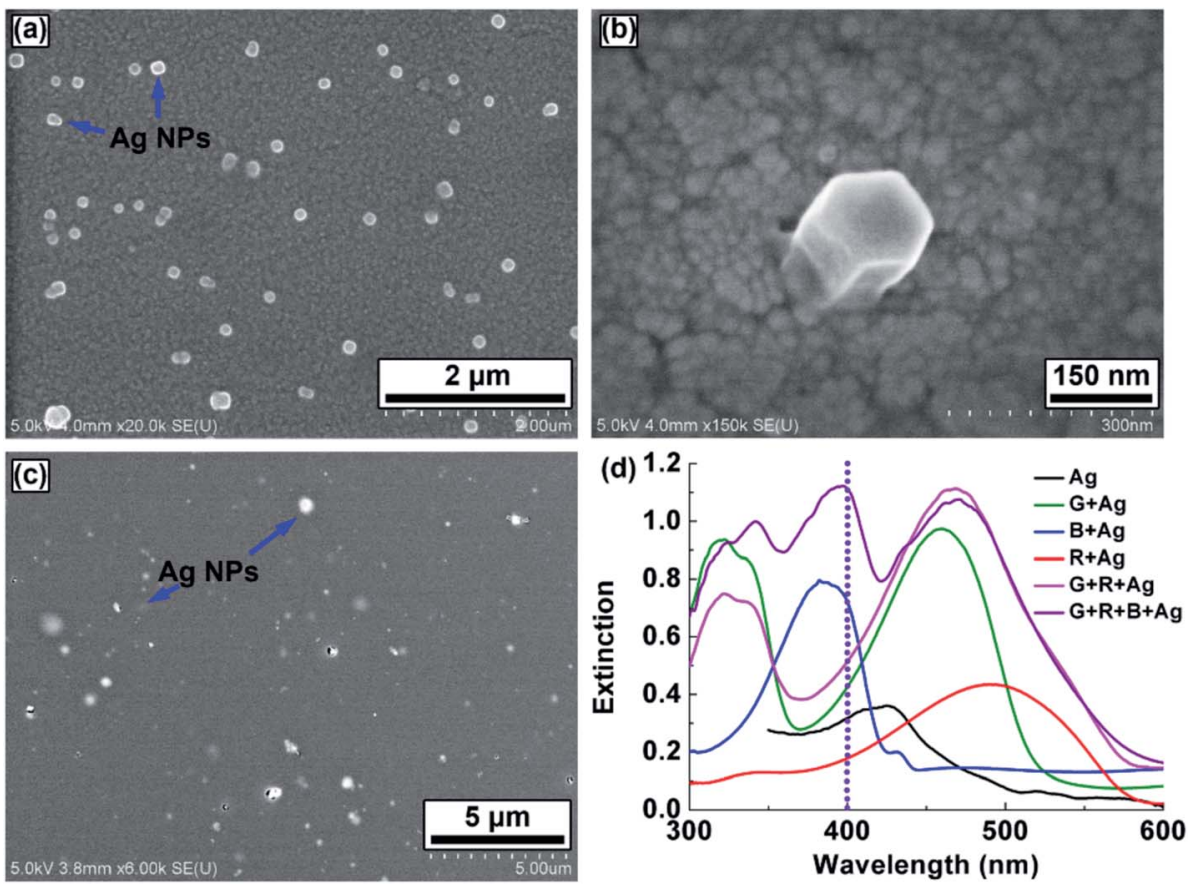

Fig. 3 SEM images of the Ag NPs (a), (b) on the indium-tin-oxide-coated glass substrate and (c) embedded in the polymer film. (d) Extinction spectra of the Ag NPs and the polymer films containing embedded Ag NPs. The purple dotted line indicates the pump beam wavelength.

attenuator to adjust its power and was focused using a lens onto the surface of the laser sample to a diameter of $1.5 \mathrm{~mm}$. Spectroscopic characterization of the RGB random laser was performed using an optical spectrometer (Maya 2000 Pro, Ocean Optics). Fig. 4(a) shows the measured emission spectra of the RGB random laser on the fiber tip. Three random lasing peaks are present in the emission spectra at $466 \mathrm{~nm}, 572 \mathrm{~nm}$, and $638 \mathrm{~nm}$, and this implies that the PVA spacers inhibit optical coupling between the different waveguides. The five-layer structure is thus approximated as a linear combination of the red, green, and blue random lasers. Fig. 4(b) shows the FWHM and output intensity values of the RGB random laser as functions of the pump intensity. The FWHM values of the three lasing peaks are all less than $10 \mathrm{~nm}$. The thresholds, which are indicated by arrows in Fig. 4(b), are located at $10 \mu \mathrm{J} \mathrm{cm}{ }^{-2}, 60 \mu \mathrm{J} \mathrm{cm}^{-2}$, and $50 \mu \mathrm{J} \mathrm{cm}^{-2}$ for the blue, green, and red components, respectively.
The mixed colors of the RGB random laser can be tuned dynamically by increasing the pump power density, as indicated by the evolution of the emission spectra shown in Fig. 4(a). The chromaticity of each emission spectrum can be calculated directly and is determined by both the emission wavelengths and the FWHM values of the RGB random lasing peaks. The locations of the mixed colors on the International Commission on Illumination (CIE) 1931 color diagram are therefore as shown in Fig. 5. There is a one-to-one correlation between the eight emission spectra and the eight black dots shown on the CIE chromaticity diagram. The blue arrow shows the evolution of the CIE coordinates with increasing pump power density, thus indicating the extended chromaticity range of the RGB random laser fabricated on the fiber tip.

There are several advantages to integrating the plasmonic random laser on a fiber facet. Firstly, the area of the laser device
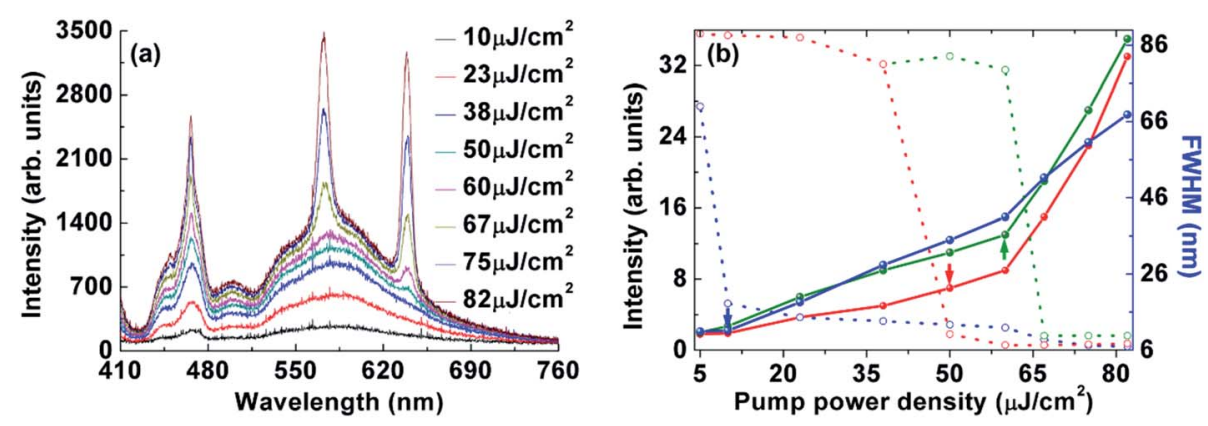

Fig. 4 (a) Emission spectra of RGB random laser fabricated on the fiber facet measured at various pump intensities. (b) FWHM and output intensity values of the RGB random laser as functions of the pump intensity. The RGB random lasing thresholds are indicated by the red, green, and blue arrows, respectively. 


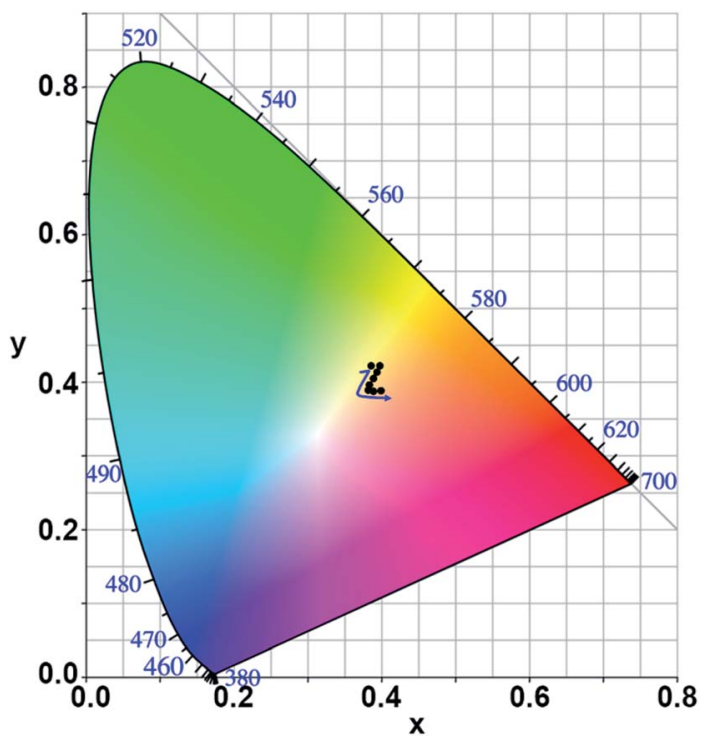

Fig. 5 Chromaticity of the RGB random laser emission spectra shown in Fig. 4(a), as indicated by the eight black dots. The blue arrow indicates the evolution of the $\mathrm{CIE}$ coordinates with increasing pump power.

is reduced by two orders of magnitude compared with that before integrating in ref. 16. Secondly, it proves that the plasmonic random laser can work stably even if the size of the laser reduces to $1 \mathrm{~mm}^{2}$. Thirdly, the dip-coating method is very simple and the fabricated laser device is robust enough for practical applications. Lastly, the plasmonic random laser on the fiber facet could have many potential applications from remote optical sensing to flexible light sources.

In conclusion, a RGB plasmonic random laser has been fabricated on the end facet of an optical fiber using a simple dip-coating method. The random laser comprised three polymer layers and two spacer layers. The total laser device thickness was approximately $3600 \mathrm{~nm}$. Ag NPs were embedded in the polymer layers to enhance optical pumping and provide strong scattering. Under appropriate optical pumping conditions, lowthreshold RGB random lasing was obtained on the optical fiber end facet.

\section{Conflicts of interest}

There are no conflicts to declare.

\section{Acknowledgements}

The authors gratefully acknowledge the financial support of the National Natural Science Foundation of China (11474014, and 11274031).

\section{References}

1 G. Dice, S. Mujumdar and A. Elezzabi, Appl. Phys. Lett., 2005, 86, 131105.
2 O. Popov, A. Zilbershtein and D. Davidov, Appl. Phys. Lett., 2006, 89, 191116.

3 X. Meng, K. Fujita, S. Murai, T. Matoba and K. Tanaka, Nano Lett., 2011, 11, 1374-1378.

4 T. Nakamura, T. Hosaka and S. Adachi, Opt. Express, 2011, 19, 467-475.

5 L. Yang, G. Feng, J. Yi, K. Yao, G. Deng and S. Zhou, Phys. Rev. A, 2011, 50, 1816-1821.

6 Z. Wang, X. Meng, S. H. Choi, S. Knitter, Y. L. Kim, H. Cao, V. M. Shalaev and A. Boltasseva, Nano Lett., 2016, 16, 24712477.

7 L. Wang, Y. Wan, L. Shi, H. Zhong and L. Deng, Opt. Express, 2016, 24, 17593-17602.

8 D. He, W. Bao, L. Long, P. Zhang, M. Jiang and D. Zhang, Opt. Laser Technol., 2017, 91, 193-196.

9 X. Meng, K. Fujita, S. Murai and K. Tanaka, Phys. Rev. A, 2009, 79, 053817.

10 E. Heydari, R. Flehr and J. Stumpe, Appl. Phys. Lett., 2013, 102, 133110.

11 Q. Qiao, C. Shan, J. Zheng, H. Zhu, S. Yu, B. Li, Y. Jia and D. Shen, Nanoscale, 2013, 5, 513-517.

12 X. Meng, K. Fujita, Y. Moriguchi, Y. Zong and K. Tanaka, Adv. Opt. Mater., 2013, 1, 573-580.

13 J. Ziegler, M. Djiango, C. Vidal, C. Hrelescu and T. A. Klar, Opt. Express, 2015, 23, 15152-15159.

14 X. Shi, Y. Wang, Z. Wang, Y. Sun, D. Liu, Y. Zhang, Q. Li and J. Shi, Appl. Phys. Lett., 2013, 103, 023504.

15 Y. Wang, X. Shi, Y. Sun, R. Zheng, S. Wei, J. Shi, Z. Wang and D. Liu, Opt. Lett., 2014, 39, 5-8.

16 T. Zhai, Z. Xu, S. Li and X. Zhang, Opt. Express, 2017, 25, 2100-2106.

17 C. J. D. Matos, L. D. S. Menezes, A. M. Brito-Silva, M. A. M. Gámez, A. S. Gomes and C. B. D. Araújo, Phys. Rev. Lett., 2007, 99, 153903.

18 M. Gagné and R. Kashyap, Opt. Express, 2009, 17, 1906719074.

19 I. D. Vatnik, D. V. Churkin, S. A. Babin and S. K. Turitsyn, Opt. Express, 2011, 19, 18486-18494.

20 W. L. Zhang, Y. J. Rao, J. M. Zhu, Z. X. Wang, Z. N. Yang and X. H. Jia, Opt. Express, 2012, 20, 14400-14405.

21 Z. N. Wang, Y. J. Rao, H. Wu, P. Y. Li, Y. Jiang, X. H. Jia and W. L. Zhang, Opt. Express, 2012, 20, 17695-17700.

22 Z. Hu, Q. Zhang, B. Miao, Q. Fu, G. Zou, Y. Chen, Y. Luo, D. Zhang, P. Wang, H. Ming and Q. Zhang, Phys. Rev. Lett., 2012, 109, 253901.

23 W. L. Zhang, Y. Y. Zhu, Y. J. Rao, Z. N. Wang, X. H. Jia and H. Wu, Opt. Express, 2013, 21, 8544-8549.

24 H. Zhijia, L. Yunyun, G. Pengfei, J. Haiming, C. Jingjing, J. Saihua and X. Kang, J. Opt., 2015, 17, 125403.

25 S. A. Babin, A. E. El-Taher, P. Harper, E. V. Podivilov and S. K. Turitsyn, Phys. Rev. A, 2011, 84, 021805.

26 S. Li, L. Wang, T. Zhai, L. Chen, M. Wang, Y. Wang, F. Tong, Y. Wang and X. Zhang, Opt. Express, 2016, 24, 12748-12754.

27 T. Y. Dong, W. T. Chen, C. W. Wang, C. P. Chen, C. N. Chen, M. C. Lin, J. M. Song, I. G. Chen and T. H. Kao, Phys. Chem. Chem. Phys., 2009, 11, 6269-6275. 